\title{
Plan for Demonstration of Online Monitoring for the Light Water Reactor Sustainability Online Monitoring Project
}

Magdy S. Tawfik

Vivek Agarwal

Nancy J. Lybeck

September 2011

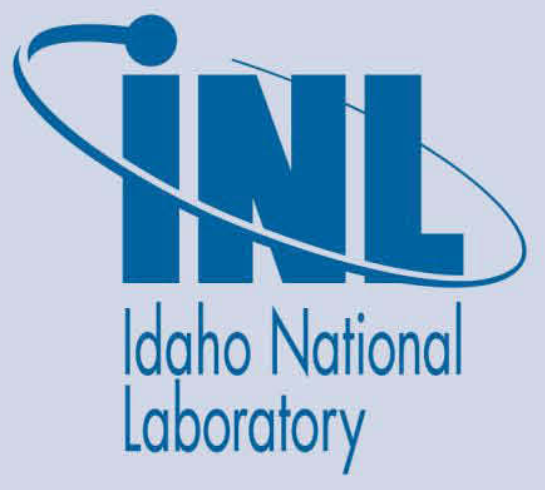

The INL is a U.S. Department of Energy National Laboratory operated by Battelle Energy Alliance 


\section{DISCLAIMER}

This information was prepared as an account of work sponsored by an agency of the U.S. Government. Neither the U.S. Government nor any agency thereof, nor any of their employees, makes any warranty, expressed or implied, or assumes any legal liability or responsibility for the accuracy, completeness, or usefulness, of any information, apparatus, product, or process disclosed, or represents that its use would not infringe privately owned rights. References herein to any specific commercial product, process, or service by trade name, trade mark, manufacturer, or otherwise, does not necessarily constitute or imply its endorsement, recommendation, or favoring by the U.S. Government or any agency thereof. The views and opinions of authors expressed herein do not necessarily state or reflect those of the U.S. Government or any agency thereof. 


\title{
Plan for Demonstration of Online Monitoring for the Light Water Reactor Sustainability Online Monitoring Project
}

\author{
Magdy S. Tawfik \\ Vivek Agarwal \\ Nancy J. Lybeck
}

September 2011

Idaho National Laboratory

Idaho Falls, Idaho 83415

http://www.inl.gov

Prepared under Work Package L-11N060203

Prepared for the

U.S. Department of Energy

Office of Nuclear Energy

Under DOE Idaho Operations Office

Contract DE-AC07-05ID14517 



\begin{abstract}
Use of online monitoring technologies and the development of diagnostic and prognostic methodologies have drawn tremendous interest in the nuclear industry. It has become important to identify and resolve problems with structures, systems, and components to ensure plant safety, efficiency, and immunity to accidents in the aging fleet of reactors. The Machine Condition Monitoring (MCM) test bed at INL will be used to demonstrate the effectiveness of advancements in online monitoring, sensors, diagnostic and prognostic technologies on a pilot-scale plant that mimics the hydraulics of a nuclear plant. As part of this research project, INL will research available prognostics architectures and their suitability for deployment in a nuclear power plant. In addition, INL will provide recommendations to improve the existing diagnostic and prognostic architectures based on the experimental analysis performed on the MCM test bed.
\end{abstract}




\section{CONTENTS}

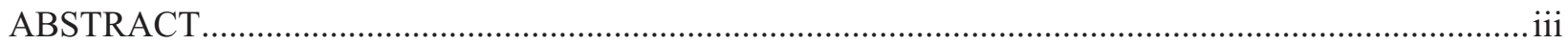

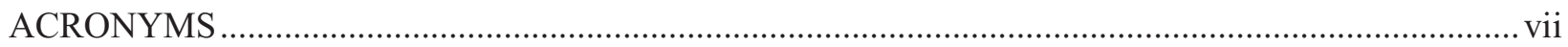

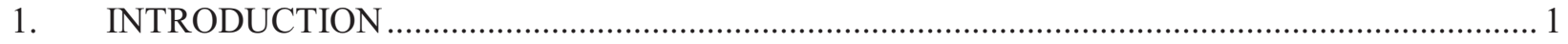

2. MACHINE CONDITION MONITORING TEST BED ….................................................... 1

2.1 The MCM Mechanical Configuration.......................................................................... 1

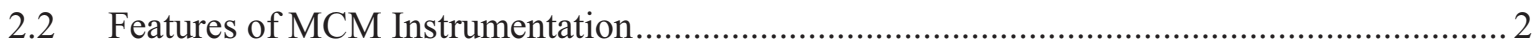

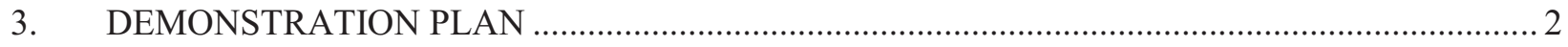

3.1 Sensor Validation or Sensor Calibration......................................................................... 2

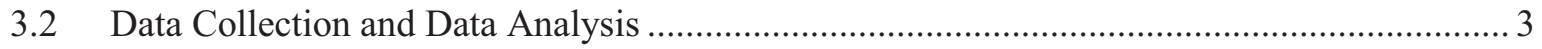

3.3 Impact on the Loop due to Individual Component Failure ................................................. 3

3.4 System Operation and Aging Issue under Degraded Power Quality .................................... 4

3.5 Physics-of-Failure Theories and Assumptions................................................................. 5

3.6 Data-Driven Diagnostic and Prognostic Algorithm Modules ............................................. 5

3.7 Experimentation to Validate Software .......................................................................... 6

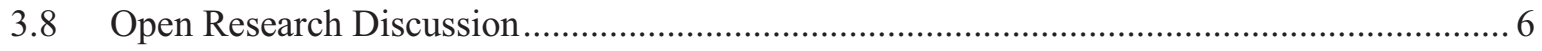

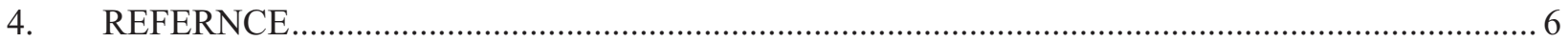

\section{FIGURES}

Figure 1. Lifecycle prognosis architecture for active critical components using the MCM test bed........... 1

Figure 2. Machine condition monitoring (MCM) test bed. (a) Overall test bed, (b) data acquisition system, and (c) active components...............................................................................

Figure 3. Data collection and data analysis module of the prognostic architecture ................................... 3

Figure 4. Diagnosis and Prognosis Modules of the Prognostic Architecture [INL] . ................................ 5 


\section{ACRONYMS}

CPVC Chlorinated Polyvinyl Chloride

HP Horse Power

INL Idaho National Laboratory

MCM Machine Condition Monitoring

NPP Nuclear Power Plant

PHM Prognostic Health Management

PLC Programmable Logic Controllers

RUL Remaining Useful Life 


\section{Plan for Demonstration of Online Monitoring for the Light Water Reactor Sustainability Online Monitoring Project}

\section{INTRODUCTION}

The Machine Condition Monitoring (MCM) test bed at INL presents the opportunity to develop and demonstrate a prognostic monitoring system on a pilot-scale plant that mimics the hydraulics of a nuclear plant service water system (at a 1/400 scale). The MCM test bed configuration and instrumentation are described in Section 1. Section 2 outlines the demonstration of a prognostic monitoring system on the MCM (illustrated in Figure 1) as a part of the Lifecycle Prognostic Architecture project, which is funded by the Light Water Reactor Sustainability program.

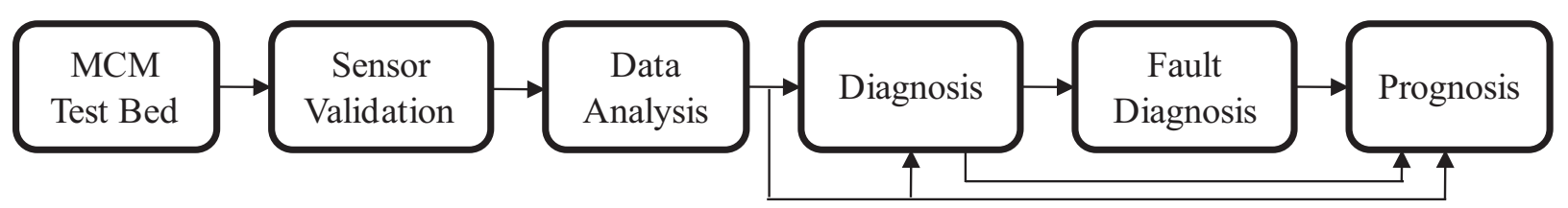

Figure 1. Lifecycle prognosis architecture for active critical components using the MCM test bed.

\section{MACHINE CONDITION MONITORING TEST BED}

The MCM test bed seen in Figure 2 consists of two hydraulic loops with pumps, motors, valves, instrumentation, data acquisition, and control. A variety of faults can be introduced to the system, including sensor and actuator faults, process faults, and control logic faults.
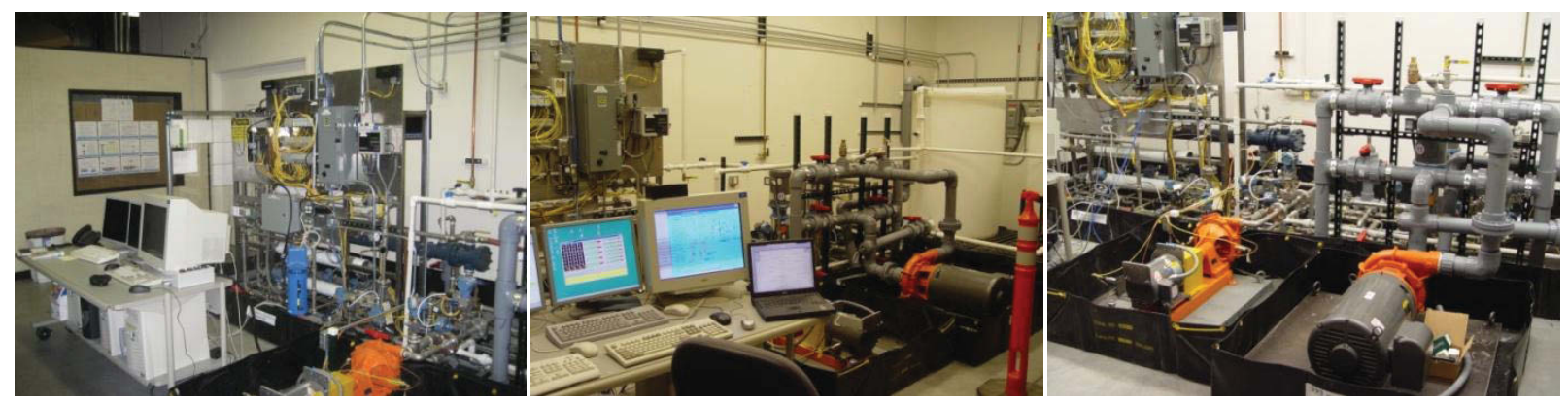

Figure 2. Machine condition monitoring (MCM) test bed. (a) Overall test bed, (b) data acquisition system, and (c) active components.

\subsection{The MCM Mechanical Configuration}

- 1-in. steel loop and 3-in. CPVC loop

- Three 5-HP pumps in small loop

- $\quad$ One 50-HP pump in large loop

- Variable frequency drive controller

- Flow, pressure, temperature, position, force instrumentation 
- Manual valves for configuring loop flows

- Motorized valves in both loops

- Instrumented butterfly valve

- Tanks in loops for flow-through, source, or drain schemes

\subsection{Features of MCM Instrumentation}

- Loop instrumentation, motor controllers, and motorized valves are connected through data I/O devices for computer display and control:

- Allen-Bradley PLC with RSView

- National Instruments LabVIEW and SignalExpress.

- Engineering, programming, and technical support are available at INL to modify and run the test bed.

- Test bed can be used in its current configuration or it can be modified.

- Test bed computers can support other commercial or custom software packages that run in a Microsoft ${ }^{\circledR}$ Windows ${ }^{\mathrm{TM}}$ environment.

\section{DEMONSTRATION PLAN}

The overarching goal of this research is to demonstrate the use of a diagnostic/prognostic system in an environment that is representative of a light water reactor. Deployment of online monitoring and prognostics on any scale requires the use of a prognostics architecture: a software product or suite of products that is necessary to perform online condition monitoring and diagnosis in addition to prognostics [INL]. As part of this ongoing research effort, INL is researching available prognostics architectures and their suitability for deployment in a nuclear power plant. At least one architecture will be used in this pilot-scale demonstration. The activities outlined below will help evaluate and enhance the chosen architecture by:

1. Identifying limitations of the software and proposing necessary upgrades in the software.

2. Developing new algorithms, as needed, which can be incorporated in the selected software as per research requirements.

The following research activities will be conducted using the MCM test bed:

\subsection{Sensor Validation or Sensor Calibration}

Different types of sensors will be installed on the MCM test bed (also referred as a test loop) in its current configuration to measure physical quantities, such as temperature, vibration, acoustics, etc. The measured signals (data) will be used to research active component health in terms of degradation over time, stressors, and failure precursors. Before and after installation of sensors, it is pertinent to perform sensor validation or sensor calibration to prevent false operation of sensors and to ensure data collection with higher confidence. Most condition-based monitoring is based on the assumption that installed sensors operate correctly, without validating the sensors. In addition, some Prognostic Health Management (PHM) algorithms are unable to distinguish the deviation in the measured signal that may be due to a fault in the sensor rather than component health degradation. 
In sensor validation procedures, sensor data is compared with reference criteria that are provided by the sensor manufacturer. Any deviation in the sensor reading from the reference criteria is referred to as a "sensor error." The sensor error should be within the permissible limit (i.e., determined either by the manufacturer or based on research objectives) for the sensor to be used for data collection. Sensors that do not perform within these limits should be replaced. Over a period of time, the performance (in terms of accuracy) of installed sensors tends to degrade and sensor errors increase. Therefore, in order to monitor the performance of installed sensors, the following steps shall be followed:

1. Test the selected software for capability to detect faulty sensor operation.

2. Develop a state estimation technique for sensor validation that will allow the health of the installed sensors to be tracked.

\subsection{Data Collection and Data Analysis}

Real-time data (signals) will be collected from installed sensors and loop instrumentation using National Instruments LabVIEW and SignalExpress Software. The measured signals may include temperature, vibration, acoustic, electrical current, etc., depending on the combination of sensors installed on active critical components of the loop.

The loop has the capability of being interfaced with other commercial or custom software packages that can run in a Microsoft Windows environment. In addition, the configuration of the loop can also be modified to allow data collection under a variety of operating conditions.

Collection of measured signals will be used to create a database; each measured signal in the database will be analyzed to develop a denoising procedure to eliminate noise in the measured signal. Denoising is required as noise in the measured signal may lead to incorrect active component health/degradation analysis. The denoised signal will be used to identify the degradation pattern and failure precurors. Figure 3 represents the data collection and data analysis module of the prognostic architecture.

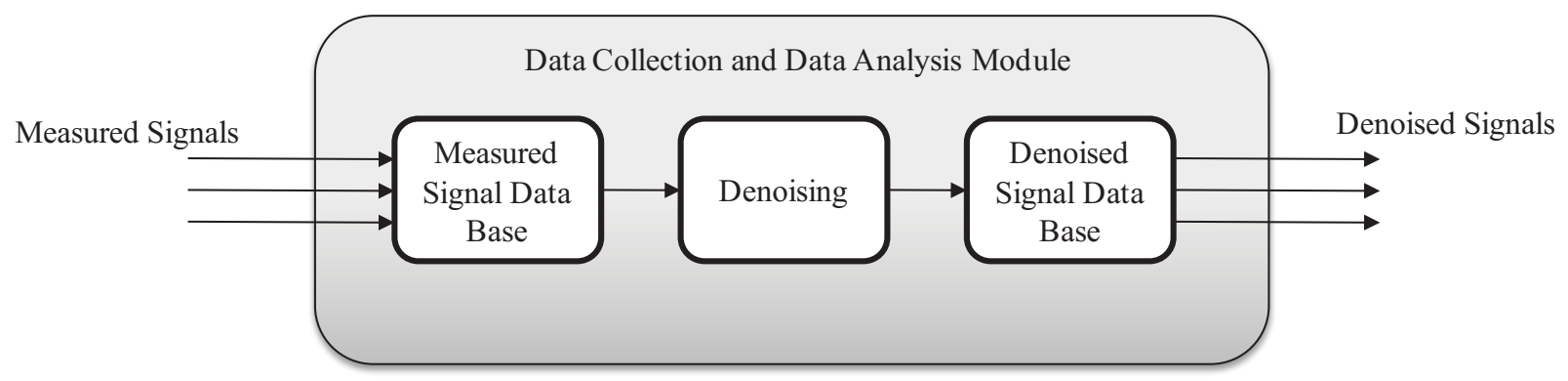

Figure 3. Data collection and data analysis module of the prognostic architecture.

\subsection{Impact on the Loop due to Individual Component Failure}

Each active component in the loop can malfunction and fail in multiple ways. The types of failures that can be observed in active components can be gearbox failure, shaft failure, motor failure, bearing failure, and so on. Malfunctioning or failure of an individual active component in the loop impacts the operational profile of other active components connected to it either directly or indirectly. The impact of that change might adversely affect some components, while others might not be affected at all. This might give rise to one or more additional faults. Any subsequent faults may be different from the original fault in terms of not only severity and criticality, but also in terms of propagation dynamics. It is possible that the original fault might not be as critical in and of itself, but the secondary faults may be detrimental. The 
progression of a fault condition and its effect on other components is largely specific to the system and will be researched using the MCM test bed. The progression of a fault condition within a component is known as fault progression and its spread to other components is known as fault propagation.

Both fault progression and fault propagation can cause a cascading effect, which can impact overall loop reliability, eventually leading to loop (system) failure. Therefore, it is important to detect and isolate faults to mitigate collateral damage. The MCM test bed will be used to characterize the inter-component operational profile dependency, which in turn will allow researchers to:

1. Characterize and identify the active component operation profile variation and its rate of variation when connected to a malfunctioning or aging component.

2. Characterize and isolate the inter-component profile variation due to different types of faults.

3. Develop a modular framework that can distinguish the fault progression and/or fault propagation within a system.

4. Identify a list of faults or combination of faults that have significant impact on the inter-component operation profile.

5. Develop a procedure that will allow identification of salient features that are due to inter-component operational dependency.

6. Recommend software upgrades based on research observations.

\subsection{System Operation and Aging Issue under Degraded Power Quality}

All of the active components are powered via an external power source. Reliable operation of an active component or system can be impacted due to degraded power quality (i.e., degraded terminal voltage). The terminal voltage or power quality depends on several factors including phase magnitude, angle, and frequency, as well as the duration of any deviation from the rated terminal voltage. Degradation in power quality can reduce the operating lifetime of active components due to variations in the operating conditions of the components.

Degradation in power quality can occur due to interconnection of renewable energy sources to the nuclear power plant (NPP). Alternatively, power degradation can be due to an intentional cyber security breach. This introduces variations in the terminal supply voltage in the form of variations in operating frequency due to harmonics, voltage sag and swell, flicker, etc. The operation of active components using degraded power quality introduces changes in the operational profile and can cause component degradation, especially in aged or aging components. As such, this project will:

1. Study the impact of degraded power quality on individual component and inter-component operational profiles.

2. Examine the impact of degraded power quality on the aging behavior of a component and overall system operation.

3. Identify any unique signal feature that indicates malfunctioning of active components due to degraded power quality.

4. Identify and classify the type of variation in the terminal voltage. 


\subsection{Physics-of-Failure Theories and Assumptions}

Physics-of-failure models are first principle models that provide a greater understanding of the mechanisms by which systems and components may fail. However, physics-of-failure model performance is restricted based on the assumptions made during development of the model. In addition, physics-offailure models are developed only for a single component with a single fault type.

In this research, a physics-of-failure model for prognosis of the MCM test bed will be developed and validated. In addition, commercial software will be used to simulate component failure for a single fault type, and the resulting prediction will be compared to the prediction obtained using the model. Based on simulation results, the physics-of-failure model for prognosis will be updated by modifying or relaxing certain assumptions made during initial model development. The updated model will then be used to predict the remaining useful life (RUL) of components for different faults types.

\subsection{Data-Driven Diagnostic and Prognostic Algorithm Modules}

This portion of the research has two modules: (1) the Diagnosis Module, and (2) the Prognosis Module.

The Diagnosis Module is the first step in identifying and classifying any type of anomalies in the behavior of active components in the loop. It has two modules, as shown in Figure 4: (i) the monitoring module, and (ii) the fault detection module. Data from the data analysis module is input into the monitoring model to predict component and system condition behavior. The predicted operational profile is compared with the data to determine the presence or occurrence of any type of fault in the active components. Any form of deviation in the operational profile from the required behavior is detected and diagnosed for the presence of a fault. The result of fault diagnosis will be used for fault isolation action as well as being used by the prognostic module to compute the RUL of the component.

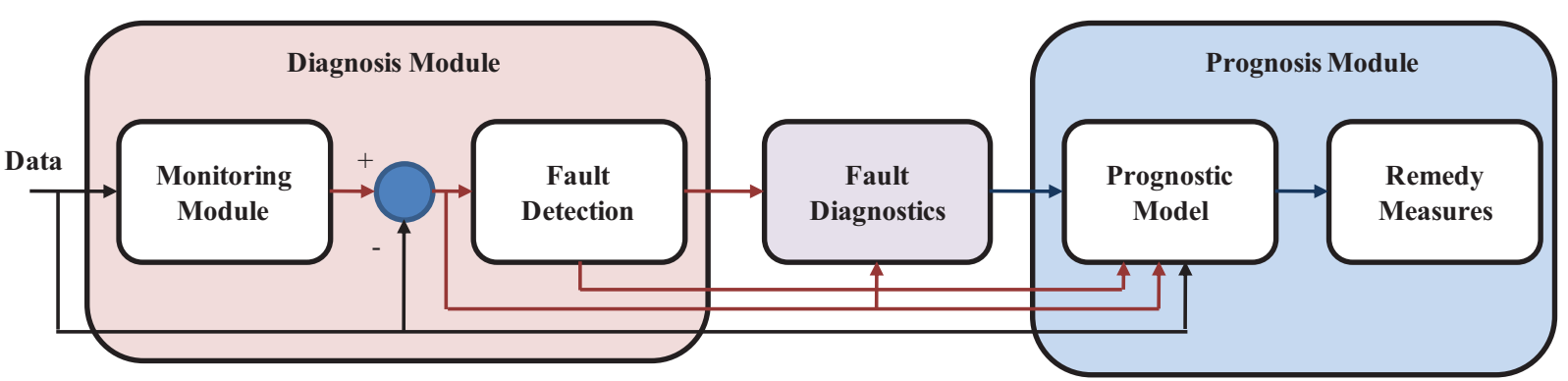

Figure 4. Diagnosis and Prognosis Modules of the Prognostic Architecture [INL].

The Prognosis Module of the software consists of prognostic models used to determine the RUL of the fault-diagnosed components under a given operating condition. Based on RUL prediction, appropriate remedial measure is suggested by the Remedy Measures Module. Prognosis models comprise both datadriven and physics-of-failure models. One of the challenges in prognostic modeling is the prediction of RUL under changing operating conditions that may arise due to fault progression and propagation.

A major research objective while validating the diagnostic and prognostic models of potential commercial software is to determine whether that software can:

1. Adapt to different types of signals from the sensors.

2. Identify different types of faults. 
3. Identify inter-component operational dependency signatures, such as fault progression and propagation.

4. Identify the deviation in operational behaviors under degraded power quality.

5. Be updated to include new algorithms for diagnosis and/or prognosis.

6. Incorporate data fusion or ensemble techniques for both to improve diagnosis and prognosis models.

7. Estimate the RUL of a component/system under a changing operating condition.

The above research objectives are aimed to provide highly accurate modeling of a complex system with low false alarm rates and highly accurate prediction of component degradation.

\subsection{Experimentation to Validate Software}

Prognosis is performed on a component to assess its RUL. Most prognosis algorithms (either data- or physics-of-failure-driven) are based on certain assumptions. If any assumption is violated in practice, the performance of a prognosis algorithm may deviate in an undesirable manner. Therefore, it is important to validate the prognostic algorithm's estimate of the RUL with the actual lifetime of the component. To do this, an experiment will be designed using the MCM test bed to validate the prognosis algorithm. The same will be done for diagnosis algorithms.

The outcome of this research will characterize the degree of correction of assumptions made during modeling of diagnosis and prognosis algorithms. In addition, it will allow improvement of current algorithm/software assessment of RUL by updating the assumptions.

\subsection{Open Research Discussion}

The outcome of this research on the MCM test bed will be shared with industry, universities, and other national laboratories to seek input on:

1. Loop design and its modification.

2. Prognostic architecture.

3. Computational complexity of the prognostic architecture.

4. Real-time challenges associated with the implementation of the prognostic architecture for a NPP.

5. Advancement in diagnosis and prognosis algorithms using new data-fusion-based techniques.

6. Quantification of uncertainties and error propagation.

7. Application of diagnosis/prognosis in the automation of an industrial control system.

\section{REFERNCE}

1. INL, "Lifecycle Prognostics Architecture for Selected High Cost Active Components," INL/EXT-1122915, 2011. 